\title{
On graph irregularity strength
}

\author{
Alan Frieze*, Ronald J. Gould ${ }^{\dagger}$, Michał Karoński ${ }^{\dagger}$, \\ and Florian Pfender ${ }^{\dagger}$
}

\begin{abstract}
An assignment of positive integer weights to the edges of a simple graph $G$ is called irregular if the weighted degrees of the vertices are all different. The irregularity strength, $s(G)$, is the maximal weight, minimized over all irregular assignments. In this paper we show, that $s(G) \leq c_{1} n / \delta$, for graphs with maximum degree $\Delta \leq n^{1 / 2}$ and minimum degree $\delta$, and $s(G) \leq c_{2}(\log n) n / \delta$, for graphs with $\Delta>n^{1 / 2}$, where $c_{1}$ and $c_{2}$ are explicit constants. To prove the result, we are using a combination of deterministic and probabilistic techniques.
\end{abstract}

AMS Subject Classification.(1991) $05 \mathrm{C} 78$

Keywords. irregularity strength, irregular assignment

${ }^{*}$ Department of Mathematical Sciences, Carnegie Mellon University, Pittsburgh, PA 15213, USA, Research supported in part by NSF grant CCR-9818411, alan@random.math.cmu.edu

${ }^{\dagger}$ Department of Mathematics and Computer Science, Emory University, Atlanta GA 30322, USA, rg@mathcs.emory.edu, michal@mathcs.emory.edu, fpfende@mathcs.emory.edu

${ }^{\ddagger}$ Faculty of Mathematics and Computer Science, Adam Mickiewicz University, Poznań, Poland, karonski@amu.edu.pl 


\section{Introduction:}

Perhaps the second oldest "fact" in graph theory is that in a simple graph, two vertices must have the same degree. This fact no longer holds for multigraphs. By an irregular multigraph we mean one in which each vertex has a different degree. Hence, a natural question would be: What is the least number of edges we would need to add to a graph in order to convert a simple graph into an irregular multigraph?

Another way to view this question is via an assignment of integer weights to the edges of the graph. Given a simple graph $G$ of order $n$, an assignment $f: E(G) \rightarrow\{1, \ldots, w\}=[w]$ of positive integers weights to the edges of $G$ is called irregular if the weighted degrees, $f(v)=\sum_{u \in N(v)} f(u v)$ of the vertices are all different. The irregularity strength, $s(G)$, is the maximal weight $w$, minimized over all irregular weight assignments, and is set to $\infty$ if no such assignment is possible. Clearly, $s(G)<\infty$ if and only if $G$ contains no isolated edges and at most one isolated vertex.

The irregularity strength was introduced in [3] by Chartrand et al. . The irregularity strength of regular graphs was considered by Faudree and Lehel in [4]. They showed that if $G$ is a $d$-regular graph of order $n, d \geq 2$, then $s(G) \leq\lceil n / 2\rceil+9$, and they conjectured that $s(G)=\left\lceil\frac{n+d-1}{d}\right\rceil+c$ for some constant $c$. This conjecture comes from the lower bound $s(G) \geq\left\lceil\frac{n+d-1}{d}\right\rceil$. For general graphs with finite irregularity strength, Aigner and Triesch [1] showed that $s(G) \leq n-1$ if $G$ is connected and $s(G) \leq n+1$ otherwise. Nierhoff [8] refined their method to show $s(G) \leq n-1$ holds for all graphs with finite irregularity strength, except for $K_{3}$. We will provide an improvement of both the Faudree-Lehel bound and the Aigner-Triesch-Nierhoff bound in this paper.

For a review of other results and open problems in this area we refer the reader to a survey paper by Lehel [7].

In this paper all graphs are simple of order $n$. The degree of a vertex $v$ is denoted by $d_{v}$ or $\operatorname{deg}(v)$, we shall denote the minimum degree of $G$ by $\delta$ and the maximum degree by $\Delta$. For terms not found here see [2] or [6]. Our upper bounds on $s(G)$ involve a function of $n$ and $\delta$ or both $\delta$ and $\Delta$, and are stated in the next Theorem. 
Theorem 1 Let $G$ be a graph with no isolated vertices or edges.

(a) If $\Delta \leq\left\lfloor(n / \ln n)^{1 / 4}\right\rfloor$, then $s(G) \leq 7 n\left(\frac{1}{\delta}+\frac{1}{\Delta}\right)$.

(b) If $\left\lfloor(n / \ln n)^{1 / 4}\right\rfloor+1 \leq \Delta \leq\left\lfloor n^{1 / 2}\right\rfloor$, then $s(G) \leq 60 n / \delta$.

(c) If $\Delta \geq\left\lfloor n^{1 / 2}\right\rfloor+1, \delta \geq\lceil 6 \log n\rceil$ then $s(G) \leq 336(\log n) n / \delta$.

For regular graphs, we get the following Theorem with improved constants.

Theorem 2 Let $G$ be a d-regular graph with no isolated vertices or edges.

(a) If $d \leq\left\lfloor(n / \ln n)^{1 / 4}\right\rfloor$, then $s(G) \leq 10 n / d+1$.

(b) If $\left\lfloor(n / \ln n)^{1 / 4}\right\rfloor+1 \leq d \leq\left\lfloor n^{1 / 2}\right\rfloor$, then $s(G) \leq 48 n / d+1$.

(c) If $d \geq\left\lfloor n^{1 / 2}\right\rfloor+1$, then $s(G) \leq 240(\log n) n / d+1$.

Observe that both (a) and (b) give bounds of the correct order of magnitude. If $\Delta \geq\left\lceil n^{1 / 2}\right\rceil+1$ and $\delta<\lfloor 6 \ln n\rfloor$, Theorem 1 does not apply, but we can still make the following statement:

Theorem 3 Let $G$ be a graph with no isolated vertices or edges. If $n$ is sufficiently large, then $s(G) \leq 14 n / \delta^{1 / 2}$.

To explain the main technique used to prove all results let us define

$$
m_{g}=\max _{X \subseteq V(G)}\{|X|: g(v)=g(u) \text { for all } v, u \in X\},
$$

where $g$ is defined as a weight assignment, i.e., $g: E(G) \rightarrow\{1,2, \ldots, w\}=$ $[w]$, for some integer $w$. In the deterministic part of our proof (see Lemma 4) we show that $s(G) \leq 3(w+1) m_{g}$. Next, we use probabilistic tools to establish bounds on $m_{g}$. Here the idea is to assign weights to edges from the set $\{1,2\}$ or $\{1,2,3\}$, and show that for such weightings, there exist assignments with $m_{g}$ of the order $n / \delta$ or $n \log n / \delta$ (see Lemmas 7, 8 and 9). 


\section{Deterministic Lemmas}

The next two Lemmas will be fundamental to our results. Their proofs follow below.

Lemma 4 Let $G$ be a graph without isolated vertices or isolated edges. Let $g: E(G) \rightarrow[w]$ be a weight assignment. Then there exists an irregular assignment $f: E(G) \rightarrow\left\{2 m_{g}, \ldots,(3 w+1) m_{g}\right\}$.

Lemma 5 Let $G$ be a d-regular graph without isolated vertices or isolated edges. Let $g: E(G) \rightarrow[w]$ be a weight assignment. Then there exists an irregular assignment $f: E(G) \rightarrow\left[(3 w-1) m_{g}+1\right]$.

We begin with a lemma needed to prove Lemma 4. We will call a tree with at most one vertex of degree greater than two, and $k$ vertices of degree one, a generalized $k$-star.

Lemma 6 Let $G$ be a graph without isolated vertices or isolated edges. Then $G$ has a factor consisting of generalized stars of order at least three.

Proof: Let $T$ be a spanning tree of a component of $G$. Note that $|V(T)| \geq 3$ by our hypothesis. We show that $T$ can be broken into disjoint generalized stars that together span $V(T)$. Then repeating this argument on each component produces the result.

To do this we induct on $|U|$, where $U=\left\{u \in V(T) \mid \operatorname{deg}_{T}(u) \geq 3\right\}$. If $|U| \leq 1$ we are done, as $T$ is itself a generalized star. Now assume the result holds on any tree $T$ with $|U|=l \geq 1$ and suppose $T$ is a tree with $|U|=l+1$. Now root $T$ at $u \in U$ and select any vertex $v \in U, v \neq u$, such that the distance in $T$ between $u$ and $v$ is maximum over all vertices of $U$. Let $T_{v}$ be the subtree of $T$ rooted at $v$ and consider $T^{\prime}=T \backslash T_{v}$. This tree has $|U|=l$ and by the induction hypothesis, we can find generalized stars in $T^{\prime}$ that span $V\left(T^{\prime}\right)$. Further, the tree $T_{v}$ is, by our choice of $v$, a generalized star of order at least three. This star, together with the collection of stars that spans $T^{\prime}$, spans $T$, completing the proof.

Proof of Lemma 4. Denote the weight class of a vertex $v \in V(G)$ as

$$
C_{v}=\{u \in V(G): g(u)=g(v)\} .
$$


Define a new weight function $\hat{f}: E \rightarrow\left[3 m_{g} w\right]$ by $\hat{f}(e)=3 m_{g} g(e)$. Note that the weight classes are unchanged under this function. Let $\mathcal{S}$ be a generalized star factor of $G$, guaranteed by Lemma 6 . We select one generalized star $S$ from $\mathcal{S}$. Let $u$ be a vertex of maximum degree in $S$ and suppose that $S$ consists of $t$ paths rooted at $u$. Let $u_{1}, u_{2}, \ldots, u_{t}$ be the neighbors of $u$ in $S$. Consider the first branch (path) of $S$, say $v_{1}, v_{2}, \ldots, v_{r}$, where $v_{1}=u_{1}$ and $r \geq 2$ (if such a branch of $S$ exists). Now begin with the last edge $v_{r} v_{r-1}$. We change the weight of this edge as follows. Put $f\left(v_{r} v_{r-1}\right)=$ $\hat{f}\left(v_{r} v_{r-1}\right)+x$, where $x$ is selected from the set $L=\left\{0,-1, \ldots,-\left(m_{g}-1\right)\right\}$ in such a way that $f\left(v_{r}\right)$, its new weighted degree, is different from the current weighted degrees of any vertex from $C_{v_{r}} \backslash\left\{v_{r}\right\}$. Since $\left|C_{v_{r}}\right| \leq m_{g}$, it is always possible to select an appropriate $x$. We now repeat this process to the edges $v_{r-1} v_{r-2}, v_{r-2} v_{r-3}, \ldots v_{2} v_{1}$, thus making $f\left(v_{r-1}\right), f\left(v_{r-2}\right), \ldots, f\left(v_{2}\right)$ unique also. To complete the first phase, repeat the procedure on the paths emanating from $u_{2}, u_{3}, \ldots, u_{t}$, in this order.

It remains to adjust the weights of the star centered at $u$. So, we change the weights of the edges $u u_{1}, u u_{2}, \ldots, u u_{t-1}$, one by one, starting at $u u_{1}$. Let $f\left(u u_{i}\right)=\hat{f}\left(u u_{i}\right)+y_{i}$, where $y_{i}$ is chosen from the set $L^{\prime}=\left\{-m_{g},-\left(m_{g}-\right.\right.$ $\left.1), \ldots, m_{g}-1, m_{g}\right\}$, in such a way that $f\left(u_{i}\right), i=1,2, \ldots, t-1$, the new weighted degree of $u_{i}$, is different from the current weighted degrees of any vertex from $C_{u_{i}} \backslash\left\{u_{i}\right\}$ and, additionally, such that $\sum_{k=1}^{i} y_{i}$ belongs to the set $\left(L \cup\left\{-m_{g}\right\}\right) \backslash\left\{f\left(u_{t} v\right)-\hat{f}\left(u_{t} v\right)\right\}$, where $v$ is the second vertex of the path starting in $u_{t}$ (if no such vertex $v$ exists, use instead $\left(L \cup\left\{-m_{g}\right\}\right) \backslash\{0\}$ ). Now we are left with $u u_{t}$. Observe that $u$ and $u_{t}$ have different weighted degrees at this time. Now let $f\left(u u_{t}\right)=\hat{f}\left(u u_{t}\right)+x$, where $x \in L^{\prime} \backslash\left\{-m_{g}\right\}$, such that both $f(u)$ and $f\left(u_{t}\right)$ are unique in their respective classes. This is possible, since there are $2 m_{g}$ options, and $C_{u}$ and $C_{u_{t}}$ can only block $2\left(m_{g}-1\right)$ of these. Finally, repeat the process for all remaining stars $S \in \mathcal{S}$.

Now for every weight class $C_{u}$, all vertices have different weighted degrees under $f$. The weighted degrees were altered from $\hat{f}$ by total values from the range $\left\{-2 m_{g}+1, \ldots, m_{g}\right\}$, the different classes were at least $3 m_{g}$ apart from each other under $\hat{f}$, so $f$ is an irregular assignment to the set $\left\{2 m_{g}, 2 m_{g}+\right.$ $\left.1, \ldots, 3 m_{g} w+m_{g}\right\}$.

Proof of Lemma 5. Use Lemma 4 to get an irregular weight assignment $f^{\prime}: E(G) \rightarrow\left\{2 m_{g}, 2 m_{g}+1, \ldots, 3 m_{g} w+m_{g}\right\}$. Now define $f: E(G) \rightarrow$ $\left[(3 w-1) m_{g}+1\right]$ by $f(e)=f^{\prime}(e)-2 m_{g}+1$. This assignment is irregular, 
since the weighted degree of every vertex is reduced by $d\left(2 m_{g}-1\right)$.

\section{Probabilistic Lemmas}

The following two lemmas will be used to get bounds on the irregularity strength of graphs with maximal degree $\Delta \leq n^{1 / 2}$. Again, the proofs follow below.

Lemma 7 Let $G$ be a graph. If $\Delta \leq(n / \ln n)^{1 / 4}$, then $\exists g: E(G) \rightarrow\{1,2\}$ such that $m_{g} \leq \frac{n}{\delta}+\frac{n}{\Delta}$.

Lemma 8 Let $G$ be a graph. If $\Delta \leq n^{1 / 2}$, then $\exists g: E(G) \rightarrow\{1,2,3\}$ such that $m_{g} \leq 6 n / \delta$.

The next lemma is used for graphs with $\Delta>n^{1 / 2}$.

Lemma 9 Let $G$ be a graph. If $n \geq 10$ and $\delta \geq 10 \log n$, then $\exists g: E(G) \rightarrow$ $\{1,2\}$ such that $m_{g} \leq 48(\log n) n / \delta$.

Finally, we state the lemma which provides bounds on $m_{g}$, without any restrictions on vertex degrees of a graph $G$, but for sufficiently large $n$ only.

Lemma 10 Let $G$ be a graph. If $n$ is sufficiently large, then $\exists g: E(G) \rightarrow$ $\{1,2\}$ such that $m_{g} \leq 2 n / \delta^{1 / 2}$.

Since the proofs of both Lemma 7 and Lemma 9 use the same model of assigning weights to the edges, at random, we will present their proof together.

\section{Proof of Lemmas $\mathbf{7}$ and $\mathbf{9}$.}

Let $X_{v}, v \in V$ be independent random variables with uniform distibution over the interval $[0,1]$, and then for $e=u v \in E$, let

$$
g(e)=\left\{\begin{array}{ll}
2 & \text { if } X_{u}+X_{v} \geq 1 \\
1 & \text { if } X_{u}+X_{v}<1
\end{array} .\right.
$$

For the non-negative integer $y \in\left\{0,1, \ldots, d_{v}\right\}$,

$$
\operatorname{Pr}\left(g(v)=d_{v}+y\right)=\int_{x=0}^{1}\left(\begin{array}{c}
d_{v} \\
y
\end{array}\right) x^{y}(1-x)^{d_{v}-y} d x=\frac{1}{d_{v}+1} \leq \frac{1}{\delta+1} .
$$


It follows for every $y$ with $\delta \leq y \leq 2 \Delta$ and $Z_{y}=|\{v \in V: g(v)=y\}|$ that

$$
\mathbf{E}\left(Z_{y}\right) \leq \frac{n}{\delta+1}
$$

To prove Lemma 7 , we assume that $G$ is a graph with maximum degree $\Delta \leq(n / \log n)^{1 / 4}$.

We apply the Hoeffding-Azuma inequality, see e.g. Janson, Euczak and Ruciński [6]. Changing the value of an $X_{v}$ can only change the value of $Z_{y}$ by at most $\Delta+1$. It follows that for $t>0$,

$$
\operatorname{Pr}\left(Z_{y} \geq \mathbf{E}\left(Z_{y}\right)+t\right) \leq \exp \left\{-\frac{t^{2}}{2 n(\Delta+1)^{2}}\right\} .
$$

Putting $t=\frac{n}{\Delta+1}$ and using (2) we see that

$$
\operatorname{Pr}\left(Z_{y} \geq \mathbf{E}\left(Z_{y}\right)+t\right)<\frac{1}{2 \Delta},
$$

and thus

$$
\operatorname{Pr}\left(\exists y: Z_{y} \geq \frac{n}{\delta}+\frac{n}{\Delta}\right)<1,
$$

and Lemma 7 follows.

We now prove Lemma 9. We use the Markov inequality for $t, k>0$ and any event $\mathcal{E}$, to obtain

$$
\operatorname{Pr}\left(Z_{y}>t \mid \mathcal{E}\right) \leq \frac{\mathbf{E}\left(\left(\begin{array}{c}
Z_{y} \\
k
\end{array}\right) \mid \mathcal{E}\right)}{\left(\begin{array}{l}
t \\
k
\end{array}\right)} .
$$

But

$$
\mathbf{E}\left(\left(\begin{array}{c}
Z_{y} \\
k
\end{array}\right) \mid \mathcal{E}\right)=\sum_{|S|=k} \operatorname{Pr}(g(v)=y, v \in S \mid \mathcal{E}) .
$$

Now fix $S=\left\{v_{1}, v_{2}, \ldots, v_{k}\right\}$ in (5). For $v \in S$ let $N_{S}(v)=N(v) \backslash S$, and let $\mu(v)=\left|N_{S}(v)\right|$. Note that $d_{v}-\mu(v) \leq k-1$. For $v \in S$ let $\xi_{1}<\xi_{2}<\cdots<\xi_{d_{v}}$ be the values of $X_{u}, u \in N(v)$, sorted in increasing order and let $\eta_{1}<\eta_{2}<\cdots<\eta_{\mu(v)}$ be the values of $X_{u}, u \in N_{S}(v)$, also sorted in increasing order. 
Note that, in general, if $\xi_{1}<\xi_{2}<\cdots<\xi_{s}$ is the sequence of order statistic from the uniform distribution over $[0,1]$, then $\xi_{i}$ has the same distribution as $\left(Y_{1}+Y_{2}+\cdots+Y_{i}\right) /\left(Y_{1}+Y_{2}+\cdots+Y_{s+1}\right)$ where $Y_{1}, Y_{2}, \ldots, Y_{s+1}$ is a sequence of independent random variables, each having exponential distribution with mean one, see for example Ross, Theorem 2.3.1 [9].

To prove the lemma we need to show the following general statement.

Lemma 11 Let $Y_{1}, Y_{2}, \ldots, Y_{s}$ be a sequence of independent random variables, each having exponential distribution with mean one. Then for any real $a>0,0<b<1$ we have

$$
\begin{aligned}
& \left.\operatorname{Pr}\left(Y_{1}+\ldots+Y_{s} \geq(1+a) s\right)\right) \leq\left((1+a) e^{-a}\right)^{s} \\
& \left.\operatorname{Pr}\left(Y_{1}+\ldots+Y_{s} \leq(1-b) s\right)\right) \leq\left((1-b) e^{b}\right)^{s} .
\end{aligned}
$$

Proof:

$$
\begin{aligned}
\operatorname{Pr}\left(Y_{1}+\ldots+Y_{s} \geq t\right) & \leq \operatorname{Pr}\left(e^{\lambda\left(Y_{1}+\cdots+Y_{s}-t\right)} \geq 1\right) \\
& \leq e^{-\lambda t} \mathbf{E}\left(e^{\lambda\left(Y_{1}+\cdots+Y_{s}\right)}\right) \\
& =\frac{e^{-\lambda t}}{(1-\lambda)^{s}}
\end{aligned}
$$

provided $\lambda \in(0,1)$.

So putting $t=(1+a) s$, we see that

$$
\operatorname{Pr}\left(Y_{1}+\ldots+Y_{s} \geq(1+a) s\right) \leq\left(\frac{e^{-\lambda(1+a)}}{1-\lambda}\right)^{s}=\left((1+a) e^{-a}\right)^{s}
$$

on putting $\lambda=a /(1+a)$.

A similar argument shows that

$$
\operatorname{Pr}\left(Y_{1}+\ldots+Y_{s} \leq(1-b) s\right) \leq\left((1-b) e^{b}\right)^{s},
$$

completing the proof of Lemma 11.

Let $k=\lfloor\log n\rfloor$ and

$$
\mathcal{E}=(\Theta<(16 \log n) / \delta)
$$

where

$$
\Theta=\max _{v \in V} \Theta_{v}, \text { and } \Theta_{v}=\max _{0 \leq i \leq d_{v}-2 k+1} \xi_{i+2 k}-\xi_{i} .
$$

Here, by default, we take $\xi_{0}=0$ and $\xi_{d_{v}+1}=1$. 
Now, observe that $g(v)=y$ implies

$$
1-X_{v} \in\left[\xi_{2 d_{v}-y}, \xi_{2 d_{v}-y+1}\right] \subset\left[\eta_{2 d_{v}-y-k+1}, \eta_{2 d_{v}-y+1}\right] \subseteq\left[\xi_{2 d_{v}-y-k+1}, \xi_{2 d_{v}-y+k}\right] .
$$

In the above formula, we take $\xi_{j}=\eta_{j}=0$ for $j \leq 0$, and $\xi_{d_{v}+j}=\eta_{\mu(v)+j}=1$ for $j \geq 1$.

Applying Lemma 11 to the order statistics defining $\Theta$, we see that

$$
\begin{aligned}
\operatorname{Pr}(\neg \mathcal{E}) & =\operatorname{Pr}\left(\exists v \in V: \Theta_{v} \geq \frac{16 \log n}{\delta}\right) \\
& \leq n \operatorname{Pr}\left(\exists 0 \leq i \leq \Delta-2 k+1: \frac{Y_{i}+\cdots+Y_{i+2 k-1}}{Y_{1}+\cdots+Y_{\delta+1}} \geq \frac{16 \log n}{\delta}\right) \\
& \leq n \operatorname{Pr}\left(Y_{1}+\cdots+Y_{\delta+1} \leq \delta / 2\right)+n^{2} \operatorname{Pr}\left(Y_{1}+\cdots+Y_{2 k} \geq 8 k\right) \\
& \leq n\left(e^{1 / 2} / 2\right)^{\delta+1}+n^{2}\left(4 e^{-3}\right)^{2 k} \\
& \leq 1 / 10 .
\end{aligned}
$$

Further,

$$
\begin{aligned}
& \operatorname{Pr}(g(v)=y, v \in S \mid \mathcal{E}) \leq \operatorname{Pr}\left(1-X_{v_{i}} \in\left[\eta_{2 d_{v_{i}-y-k+1}}, \eta_{2 d_{v_{i}}-y+1}\right], i=1,2, \ldots, k \mid \mathcal{E}\right) \\
& \quad \leq 2 \operatorname{Pr}\left(1-X_{v_{i}} \in\left[\eta_{2 d_{v_{i}-y-k+1}}, \eta_{2 d_{v_{i}}-y-k+1}+\frac{16 \log n}{\delta}\right], i=1,2, \ldots, k\right) \\
& \quad \leq 2\left(\frac{16 \log n}{\delta}\right)^{k} .
\end{aligned}
$$

From (4) and (5) we obtain

$$
\operatorname{Pr}\left(\exists y: Z_{y}>t \mid \mathcal{E}\right) \leq 2 n\left(\begin{array}{l}
t \\
k
\end{array}\right)^{-1}\left(\begin{array}{l}
n \\
k
\end{array}\right)\left(\frac{16 \log n}{\delta}\right)^{k} .
$$

Putting $t=48(\log n) n \delta^{-1}$ together with (6) establishes

$$
\operatorname{Pr}\left(\exists y: Z_{y}>t\right) \leq \operatorname{Pr}\left(\exists y: Z_{y}>t \mid \mathcal{E}\right)+\operatorname{Pr}(\neg \mathcal{E})<1
$$

proving Lemma 9. 
Proof of Lemma 8. For every vertex $v$ independently assign a number $W_{v}$ from $\left\{0, \ldots, d_{v}\right\}$ uniformly at random. Now pick a random subset $N \subseteq N(v)$ of size $W_{v}$, and for every $u \in N$, set $v_{u}=1$, and for every $u \in N(v) \backslash N$, set $v_{u}=0$.

Let $g: E \rightarrow[3]$ as follows: For $u v \in E$, let $g(u v)=1+v_{u}+u_{v}$. For a vertex $v$, let $g(v)=\sum_{u \in N(v)} g(u v)$. For some integer $y$ with $\delta \leq y \leq 3 \Delta$, let $Z_{y}=|\{v \in V: g(v)=y\}|$. Then

$$
\mathbf{E}\left(Z_{y}\right) \leq \frac{n}{\delta}
$$

since

$$
\operatorname{Pr}(g(v)=y)=\operatorname{Pr}\left(W_{v}=y-d-\sum_{u \in N(v)} u_{v}\right) \leq \frac{1}{d_{v}+1} .
$$

By the symmetry of the construction we know that $\forall x \in V, v, u \in N(x)$ :

$$
\begin{aligned}
& \operatorname{Pr}\left(x_{v}=1\right)=1 / 2, \\
& \operatorname{Pr}\left(x_{v}=x_{u}=1\right)=\operatorname{Pr}\left(x_{v}=x_{u}=0\right)=1 / 3, \\
& \operatorname{Pr}\left(x_{v}=1, x_{u}=0\right)=\operatorname{Pr}\left(x_{v}=0, x_{u}=1\right)=1 / 6 .
\end{aligned}
$$

To use Chebyshev's inequality, we have to bound the variance of $Z_{y}$ :

$$
\operatorname{Var}\left(Z_{y}\right)=\sum_{v \in V} \sum_{u \in V}(\operatorname{Pr}(g(v)=g(u)=y)-\operatorname{Pr}(g(v)=y) \operatorname{Pr}(g(u)=y)) .
$$

Fix a $v \in V$, and consider

$$
S_{v}=\sum_{u \in V}(\operatorname{Pr}(g(v)=g(u)=y)-\operatorname{Pr}(g(v)=y) \operatorname{Pr}(g(u)=y)) .
$$

Divide $V$ into three classes $V_{1}, V_{2}, V_{3}$, and consider the partial sums

$$
S_{i}=\sum_{u \in V_{i}}(\operatorname{Pr}(g(v)=g(u)=y)-\operatorname{Pr}(g(v)=y) \operatorname{Pr}(g(u)=y)) .
$$

Class 1: $V_{1}=\{v\}$.

$$
S_{1} \leq \operatorname{Pr}(g(v)=y) \leq \frac{1}{d_{v}} \leq \frac{\Delta}{\delta^{2}}
$$


Class 2: $V_{2}=N(v)$.

$$
\begin{aligned}
S_{2} & \leq d_{v} \operatorname{Pr}(g(v)=g(u)=y) \\
& \leq d_{v} \operatorname{Pr}\left(W_{v}=y-d_{v}-\sum_{x \in N(v)} x_{v} \mid g(u)=y\right) \operatorname{Pr}\left(W_{u}=y-d_{u}-\sum_{x \in N(u)} x_{u}\right) \\
& \leq d_{v} \frac{2}{\left(d_{v}+1\right)} \frac{1}{\left(d_{u}+1\right)}<\frac{2}{d_{u}} \leq \frac{2 \Delta}{\delta^{2}} .
\end{aligned}
$$

Class 3: $V_{3}=V \backslash(\{v\} \cup N(v))$.

Let $u \in V_{3}$, and let $c=|N(v) \cap N(u)|$. For the sake of the analysis, pick a random subset $\mathcal{A}$ from $\left\{x \in N(u) \cap N(v): x_{u}=x_{v}\right\}$, by choosing each vertex with probability $1 / 2$. So, using (8), for every vertex $x \in N(u) \cap N(v)$,

$$
\begin{aligned}
\operatorname{Pr}\left(x_{u}=x_{v}=1 \wedge x \in \mathcal{A}\right)=\operatorname{Pr}\left(x_{u}=x_{v}=1 \wedge x \notin \mathcal{A}\right)= \\
\operatorname{Pr}\left(x_{u}=x_{v}=0 \wedge x \in \mathcal{A}\right)=\operatorname{Pr}\left(x_{u}=x_{v}=0 \wedge x \notin \mathcal{A}\right)= \\
\operatorname{Pr}\left(x_{u}=0 \wedge x_{v}=1\right)=\operatorname{Pr}\left(x_{u}=1 \wedge x_{v}=0\right)=1 / 6
\end{aligned}
$$

and

$$
\operatorname{Pr}(x \in \mathcal{A})=1 / 3 .
$$

Let $A \subseteq N(u) \cap N(v)$, and let $a=|A|$.

Then, for every vertex $x \in N(u) \cap N(v)$,

$$
\begin{aligned}
\operatorname{Pr}\left(x_{u}=x_{v}=1 \mid \mathcal{A}=A \wedge x \notin A\right)=\frac{\operatorname{Pr}\left(x_{u}=x_{v}=1 \wedge \mathcal{A}=A \mid x \notin A\right)}{\operatorname{Pr}(\mathcal{A}=A \mid x \notin A)} & = \\
\frac{(1 / 6)(1 / 3)^{a}(2 / 3)^{c-a-1}}{(1 / 3)^{a}(2 / 3)^{c-a}} & =\frac{1}{4} .
\end{aligned}
$$

By symmetry, we get

$$
\begin{aligned}
\operatorname{Pr}\left(x_{u}=x_{v}=0 \mid \mathcal{A}=A\right)=\operatorname{Pr}\left(x_{u}=\right. & \left.0, x_{v}=1 \mid \mathcal{A}=A\right)= \\
& \operatorname{Pr}\left(x_{u}=1, x_{v}=0 \mid \mathcal{A}=A\right)=1 / 4 .
\end{aligned}
$$

Thus, given $x \notin A$ and $\mathcal{A}=A$, the events $\left(x_{v}=1\right)$ and $\left(x_{u}=1\right)$ are independent. For $x \in A$, we get

$\operatorname{Pr}\left(x_{u}=x_{v}=1 \mid \mathcal{A}=A \wedge x \in A\right)=\operatorname{Pr}\left(x_{u}=x_{v}=0 \mid \mathcal{A}=A \wedge x \in A\right)=1 / 2$. 
We introduce the following notation:

$$
\begin{aligned}
P_{A} & =\operatorname{Pr}(g(v)=g(w)=y \mid \mathcal{A}=A)-\operatorname{Pr}(g(v)=y \mid \mathcal{A}=A) \operatorname{Pr}(g(w)=y \mid \mathcal{A}=A) \\
& =\operatorname{Pr}(g(v)=g(w)=y \mid \mathcal{A}=A)-\operatorname{Pr}(g(v)=y) \operatorname{Pr}(g(w)=y),
\end{aligned}
$$

since $\operatorname{Pr}(g(v)=y)$ is independent from the choice of $\mathcal{A}$. In particular,

$$
P_{\emptyset}=\operatorname{Pr}(g(v)=g(w)=y \mid \mathcal{A}=\emptyset)-\operatorname{Pr}(g(v)=y) \operatorname{Pr}(g(w)=y)=0 .
$$

For $A \neq \emptyset$, pick any $x \in A$. We want to bound the difference $P_{A}-P_{A \backslash x}$. Let

$$
b_{v}=d_{v}+\sum_{z \in N(v) \backslash x} z_{v}, b_{u}=d_{u}+\sum_{z \in N(u) \backslash x} z_{u} .
$$

Now consider the difference between $P_{A}$ and $P_{A \backslash x}$, given that $b_{v}=l$ and $b_{u}=r$, and denote it by

$$
\begin{aligned}
P_{A}^{l, r}-\quad & P_{A \backslash x}^{l, r}= \\
=\quad & \operatorname{Pr}\left(g(v)=g(w)=y \mid \mathcal{A}=A \wedge b_{v}=l \wedge b_{u}=r\right) \\
- & \operatorname{Pr}\left(g(v)=g(w)=y \mid \mathcal{A}=A \backslash x \wedge b_{v}=l \wedge b_{u}=r\right) \\
=\quad & {\left[\operatorname{Pr}\left(x_{u}=x_{v}=1 \mid \mathcal{A}=A\right)-\operatorname{Pr}\left(x_{u}=x_{v}=1 \mid \mathcal{A}=A \backslash x\right)\right] } \\
& \times \operatorname{Pr}\left(W_{v}=y-l-1\right) \operatorname{Pr}\left(W_{u}=y-r-1\right) \\
+ & {\left[\operatorname{Pr}\left(x_{u}=x_{v}=0 \mid \mathcal{A}=A\right)-\operatorname{Pr}\left(x_{u}=x_{v}=0 \mid \mathcal{A}=A \backslash x\right)\right] } \\
& \times \operatorname{Pr}\left(W_{v}=y-l\right) \operatorname{Pr}\left(W_{u}=y-r\right) \\
+ & {\left[\operatorname{Pr}\left(x_{u}=1 \wedge x_{v}=0 \mid \mathcal{A}=A\right)-\operatorname{Pr}\left(x_{u}=1 \wedge x_{v}=0 \mid \mathcal{A}=A \backslash x\right)\right] } \\
& \times \operatorname{Pr}\left(W_{v}=y-l\right) \operatorname{Pr}\left(W_{u}=y-r-1\right) \\
+ & {\left[\operatorname{Pr}\left(x_{u}=0 \wedge x_{v}=1 \mid \mathcal{A}=A\right)-\operatorname{Pr}\left(x_{u}=0 \wedge x_{v}=1 \mid \mathcal{A}=A \backslash x\right)\right] } \\
& \times \operatorname{Pr}\left(W_{v}=y-l-1\right) \operatorname{Pr}\left(W_{u}=y-r\right) \\
\frac{1}{4} & {\left[\operatorname{Pr}\left(W_{v}=y-l-1\right) \operatorname{Pr}\left(W_{u}=y-r-1\right)+\operatorname{Pr}\left(W_{v}=y-l\right) \operatorname{Pr}\left(W_{u}=y-r\right)\right.} \\
& \left.-\operatorname{Pr}\left(W_{v}=y-l\right) \operatorname{Pr}\left(W_{u}=y-r-1\right)-\operatorname{Pr}\left(W_{v}=y-l-1\right) \operatorname{Pr}\left(W_{u}=y-r\right)\right] .
\end{aligned}
$$

Therefore,

$$
P_{A}^{l, r}-P_{A \backslash x}^{l, r}= \begin{cases}1 /\left[4\left(d_{v}+1\right)\left(d_{u}+1\right)\right] & \text { if }\left(r=y-d_{u}-1 \wedge l=y-d_{v}-1\right) \\ & \text { or }(r=y \wedge l=y), \\ -1 /\left[4\left(d_{v}+1\right)\left(d_{u}+1\right)\right] & \text { if }\left(r=y-d_{u}-1 \wedge l=y\right) \\ & \text { or }\left(r=y \wedge l=y-d_{v}-1\right), \\ 0 & \text { otherwise. }\end{cases}
$$


Thus, summing over all possible values of $l, r$ and

$$
\begin{aligned}
t=\mid\{z \in & \left.\mathcal{A} \backslash x: z_{u}=z_{v}=1\right\} \mid \\
P_{A}- & P_{A \backslash x} \leq \\
\leq & 1 /\left[4\left(d_{v}+1\right)\left(d_{u}+1\right)\right] \\
& \times\left[\operatorname{Pr}\left(b_{u}=y-d_{u}-1 \wedge b_{v}=y-d_{v}-1\right)+\operatorname{Pr}\left(b_{u}=y \wedge b_{v}=y\right)\right] \\
\leq & 1 /\left[4\left(d_{v}+1\right)\left(d_{u}+1\right)\right] \\
& \times\left[\sum_{t=0}^{a-1}\left(\begin{array}{c}
a-1 \\
t
\end{array}\right) 2^{-a+1}\left(\begin{array}{c}
d_{u}-a \\
y-2 d_{u}-1-t
\end{array}\right)\left(\begin{array}{c}
d_{v}-a \\
y-2 d_{v}-1-t
\end{array}\right) 2^{-d_{u}-d_{v}+2 a}\right. \\
& \left.+\sum_{t=0}^{a-1}\left(\begin{array}{c}
a-1 \\
t
\end{array}\right) 2^{-a+1}\left(\begin{array}{c}
d_{u}-a \\
y-d_{u}-t
\end{array}\right)\left(\begin{array}{c}
d_{v}-a \\
y-d_{v}-t
\end{array}\right) 2^{-d_{u}-d_{v}+2 a}\right] \\
\leq & \frac{1}{\left(d_{v}+1\right)\left(d_{u}+1\right)}\left(\begin{array}{c}
d_{u}-a \\
\left(d_{u}-a\right) / 2
\end{array}\right)\left(\begin{array}{c}
d_{v}-a \\
\left(d_{v}-a\right) / 2
\end{array}\right) 2^{-d_{u}-d_{v}+a} \sum_{t=0}^{a-1}\left(\begin{array}{c}
a-1 \\
t
\end{array}\right) .
\end{aligned}
$$

Suppose first that $1 \leq a \leq \delta / 3$. Then,

$$
\begin{aligned}
P_{A}-P_{A \backslash x} \leq \frac{2^{-d_{v}-d_{u}+2 a-1}}{\left(d_{v}+1\right)\left(d_{u}+1\right)}\left(\frac{2^{d_{v}-a+1}}{\left(d_{v}-a\right)^{1 / 2}}\right)\left(\frac{2^{d_{u}-a+1}}{\left(d_{u}-a\right)^{1 / 2}}\right)= \\
\frac{2}{\left(d_{v}+1\right)\left(d_{u}+1\right)\left(d_{v}-a\right)^{1 / 2}\left(d_{u}-a\right)^{1 / 2}} \leq \frac{3}{d_{v} \delta^{2}} .
\end{aligned}
$$

Hence,

$$
P_{A} \leq \frac{3 a}{d_{v} \delta^{2}} \leq \frac{3 c}{d_{v} \delta^{2}}
$$

Note that for all $A$,

$$
\operatorname{Pr}(g(v)=g(u)=y \mid \mathcal{A}=A) \leq \frac{1}{\left(d_{v}+1\right)\left(d_{u}+1\right)},
$$

hence, for $a>\delta / 3$,

$$
P_{A} \leq \operatorname{Pr}(g(v)=g(u)=y \mid \mathcal{A}=A) \leq \frac{3 a}{d_{v} \delta^{2}} \leq \frac{3 c}{d_{v} \delta^{2}} .
$$

Therefore, combining (11), (12) and (13),

$$
\begin{aligned}
\operatorname{Pr}(g(v)=g(u)=y)-\operatorname{Pr}(g(v)=y) \operatorname{Pr}(g(u)=y) & \leq \\
\sum_{A \subseteq N(u) \cap N(v)}\left(3 c / d_{v} \delta^{2}\right) \operatorname{Pr}(\mathcal{A}=A) & =\frac{3|N(v) \cap N(u)|}{d_{v} \delta^{2}} .
\end{aligned}
$$


Now notice that $\sum_{u \in V}|N(v) \cap N(u)|$ counts the number of walks of length two starting in $v$, thus $\sum_{u \in V}|N(v) \cap N(u)| \leq d_{v} \Delta$, and therefore

$$
S_{3} \leq \sum_{u \in V_{3}} \frac{3|N(v) \cap N(u)|}{d_{v} \delta^{2}} \leq \frac{3 \Delta}{\delta^{2}} .
$$

Altogether, we get from (9), (10) and (14),

$$
S_{v}=S_{1}+S_{2}+S_{3} \leq \frac{6 \Delta}{\delta^{2}},
$$

and thus,

$$
\operatorname{Var}\left(Z_{y}\right)=\sum_{v \in V} S_{v} \leq \frac{6 n \Delta}{\delta^{2}} .
$$

By Chebyshev's inequality and (7) we get

$$
\operatorname{Pr}\left(Z_{y}>6 n / \delta\right) \leq \frac{\operatorname{Var}\left(Z_{y}\right)}{(5 n / \delta)^{2}}<\frac{1}{3 \Delta}
$$

and thus,

$$
\operatorname{Pr}\left(\exists y: Z_{y}>6 n / \delta\right)<1,
$$

finishing the proof.

\section{Proof of Lemma 10.}

Choose $g$ randomly from $\{1,2\}^{E}$. Observe that $g(v)-d_{v}$ has the binomial distribution $B i\left(d_{v}, 1 / 2\right)$. For a non-negative integer $y$ let

$$
V_{y}=\left\{v:\left|y-\frac{3}{2} d_{v}\right| \leq\left(2 d_{v} \log n\right)^{1 / 2}\right\} .
$$

The Chernoff bounds for the tails of the binomial (see for example [6]) imply that for any $t>0$,

$$
\operatorname{Pr}\left(\left|g(v)-\frac{3}{2} d_{v}\right| \geq t\right) \leq e^{-2 t^{2} / d_{v}}
$$

Hence,

$$
\operatorname{Pr}(g(v)=y) \leq \frac{1}{n^{4}} \quad \text { if } v \notin V_{y} .
$$


Now consider $v \in V_{y}$. Clearly,

$$
\operatorname{Pr}(g(v)=y)=0 \quad \text { if } d_{v}<y / 2 .
$$

Case 1: $y \geq n^{1 / 4}$

If $d_{v} \geq y / 2 \geq n^{1 / 4} / 2$ then we can use Stirling's inequality or apply Feller [5], Chapter VII (2.7) to get

$$
\operatorname{Pr}(g(v)=y)=\frac{1}{2^{d_{v}}}\left(\begin{array}{c}
d_{v} \\
y-d_{v}
\end{array}\right) \approx \sqrt{\frac{2}{\pi d_{v}}} e^{-z^{2} / 2},
$$

where $z=2\left(y-\frac{3}{2} d_{v}\right) / d_{v}^{1 / 2}$.

Let $Z_{y}=|\{v: g(v)=y\}|$. It follows from (15), (16) and (17) that

$$
\mathbf{E}\left(Z_{y}\right) \leq \frac{\left|V_{y}\right|}{\delta^{1 / 2}}
$$

Let

$$
Z_{y}^{1}=\left|\left\{v \in V_{y}: g(v)=y\right\}\right| \text { and } Z_{y}^{2}=\left|\left\{v \notin V_{y}: g(v)=y\right\}\right| .
$$

It follows from (15) that

$$
\operatorname{Pr}\left(Z_{y}^{2} \neq 0\right) \leq \frac{1}{n^{3}}
$$

Note also that $v \in V_{y}$ implies that

$$
y=\frac{3}{2} d_{v}+O\left(\left(d_{v} \log n\right)^{1 / 2}\right) .
$$

Now for $t>0$ and $k=(\log n)^{2}$ we use the Markov inequality to obtain

$$
\operatorname{Pr}\left(Z_{y}^{1}>t\right) \leq \frac{\mathbf{E}\left(\left(\begin{array}{c}
Z_{y}^{1} \\
k
\end{array}\right)\right)}{\left(\begin{array}{l}
t \\
k
\end{array}\right)} .
$$

But

$$
\begin{aligned}
& \mathbf{E}\left(\left(\begin{array}{c}
Z_{y}^{1} \\
k
\end{array}\right)\right)=\sum_{S \subseteq V_{y},|S|=k} \operatorname{Pr}(g(v)=y, v \in S) \\
& =\sum_{S \subseteq V_{y},|S|=k} \sum_{\xi \in\{1,2\}^{E_{S}}} \operatorname{Pr}\left(g(v)=y, v \in S \mid g\left(E_{S}\right)=\xi\right) \operatorname{Pr}\left(g\left(E_{S}\right)=\xi\right)
\end{aligned}
$$


where $E_{S}=\{e \in E: e \subseteq S\}$.

Now fix $S$ in (22). For $v \in S$ let

$$
A_{v}=\{e=u v \in E: u \notin S\} \text { and } B_{v}=\{e=u v \in E: u \in S\} .
$$

Then, if $\left|g\left(B_{v}\right)\right|$ denotes $\sum_{u \in B_{v}} g(u)$,

$$
\begin{aligned}
\operatorname{Pr}\left(g(v)=y \mid g\left(E_{S}\right)=\xi\right) & =\operatorname{Pr}\left(\left|g\left(A_{v}\right)\right|=y-\left|g\left(B_{v}\right)\right|\right) \\
& =2^{-\left|A_{v}\right|}\left(\begin{array}{c}
\left|A_{v}\right| \\
y-\left|g\left(B_{v}\right)\right|-\left|A_{v}\right|
\end{array}\right) .
\end{aligned}
$$

Therefore,

$$
\begin{aligned}
& \frac{\operatorname{Pr}\left(\left|g\left(A_{v}\right)\right|=y-\left|g\left(B_{v}\right)\right|\right)}{\operatorname{Pr}(g(v)=y)}=2^{\left|B_{v}\right|} \frac{\left(\begin{array}{c}
\left.\mid \begin{array}{c}
\left|A_{v}\right| \\
y-\mid g\left(B_{v}|-| A_{v} \mid\right.
\end{array}\right) \\
\left(\begin{array}{c}
d_{v} \\
y-d_{v}
\end{array}\right)
\end{array}\right.}{=} \\
= & 2^{\left|B_{v}\right|} \frac{\left|A_{v}\right|\left(\left|A_{v}\right|-1\right) \cdots\left(2\left|A_{v}\right|+\left|g\left(B_{v}\right)\right|-y+1\right)}{1 \times 2 \times \cdots \times\left(y-\left|g\left(B_{v}\right)\right|-\left|A_{v}\right|\right)} \cdot \frac{1 \times 2 \times \cdots \times\left(y-d_{v}\right)}{d_{v}\left(d_{v}-1\right) \cdots\left(2 d_{v}-y+1\right)} .
\end{aligned}
$$

Now we use

$$
\left|A_{v}\right|+\left|B_{v}\right|=d_{v} \text { and }\left|B_{v}\right| \leq\left|g\left(B_{v}\right)\right| \leq 2\left|B_{v}\right| \leq 2 k
$$

and (20) to verify that

$$
\begin{aligned}
& \frac{1 \times 2 \times \cdots \times\left(y-d_{v}\right)}{1 \times 2 \times \cdots \times\left(y-\left|g\left(B_{v}\right)\right|-\left|A_{v}\right|\right)}= \\
& \left(y-d_{v}\right)\left(y-d_{v}-1\right) \cdots\left(y-\left|g\left(B_{v}\right)\right|-\left|A_{v}\right|+1\right)= \\
& \left(\frac{1}{2} d_{v}\right)^{\left|g\left(B_{v}\right)\right|-\left|B_{v}\right|}\left(1+O\left(k\left(\frac{\log n}{d_{v}}\right)^{1 / 2}\right)\right)
\end{aligned}
$$

and

$$
\begin{aligned}
& \frac{\left|A_{v}\right|\left(\left|A_{v}\right|-1\right) \cdots\left(2\left|A_{v}\right|+\mid g\left(\left|B_{v}\right|\right)-y+1\right)}{d_{v}\left(d_{v}-1\right) \cdots\left(2 d_{v}-y+1\right)}= \\
& \quad \frac{\left(2 d_{v}-y\right)\left(2 d_{v}-y-1\right) \cdots\left(2\left|A_{v}\right|+\left|g\left(B_{v}\right)\right|-y+1\right)}{d_{v}\left(d_{v}-1\right) \cdots\left(\left|A_{v}\right|+1\right)}= \\
& d_{v}^{\left|B_{v}\right|-\left|g\left(B_{v}\right)\right|} \times 2^{\left|g\left(B_{v}\right)\right|-2\left|B_{v}\right|}\left(1+O\left(k\left(\frac{\log n}{d_{v}}\right)^{1 / 2}\right)\right) .
\end{aligned}
$$


Plugging (25) and (26) into (24) we see that

$$
\frac{\operatorname{Pr}\left(\left|g\left(A_{v}\right)\right|=y-\left|g\left(B_{v}\right)\right|\right)}{\operatorname{Pr}(g(v)=y)}=1+O\left(k\left(\frac{\log n}{d_{v}}\right)^{1 / 2}\right) .
$$

So from (22) and (23) we see that

$$
\begin{gathered}
\mathbf{E}\left(\left(\begin{array}{c}
Z_{y}^{1} \\
k
\end{array}\right)\right) \leq \\
\sum_{S \subseteq V_{y},|S|=k} \sum_{\xi \in\{1,2\}} \prod_{E_{S}}\left(\left(1+O\left(k\left(\frac{\log n}{d_{v}}\right)^{1 / 2}\right)\right) \operatorname{Pr}(g(v)=y)\right) \operatorname{Pr}\left(g\left(E_{S}\right)=\xi\right) \\
\leq\left(1+O\left(k^{2} \frac{(\log n)^{1 / 2}}{n^{1 / 8}}\right)\right) \sum_{S \subseteq V_{y},|S|=k} \prod_{v \in S} \operatorname{Pr}(g(v)=y) \\
\leq(1+o(1)) \frac{1}{k !}\left(\sum_{S \subseteq V_{y},|S|=k} \operatorname{Pr}(g(v)=y)\right)^{k} \\
=(1+o(1)) \frac{\mathbf{E}\left(Z_{y}^{1}\right)^{k}}{k !} .
\end{gathered}
$$

So (18), (21) imply

$$
\operatorname{Pr}\left(Z_{y}^{1}>2 \frac{n}{\delta^{1 / 2}}\right) \leq(1+o(1)) \frac{\mathbf{E}\left(Z_{y}^{1}\right)^{k}}{\left(2 n / \delta^{1 / 2}\right)^{k}} \leq(1+o(1)) 2^{-k}
$$

and then together with (19) we get

$$
\operatorname{Pr}\left(\exists y: Z_{y}>2 \frac{n}{\delta^{1 / 2}}\right) \leq 2 n\left((1+o(1)) 2^{-k}+n^{-3}\right)=o(1) .
$$

Case 2: $y \leq n^{1 / 4}$.

Assume that $V_{y} \neq \emptyset$. We apply the Hoeffding-Azuma inequality. Changing the value of $g$ on a single edge can only change the value of $Z_{y}^{1}$ by at most 2. Also, $Z_{y}^{1}$ is determined by the outcome of at most

$$
\sum_{v \in V_{y}} d_{v} \leq\left|V_{y}\right|\left(y+(\log n)^{2}\right)
$$


random choices. It follows that for $t>0$,

$$
\operatorname{Pr}\left(Z_{y}^{1} \geq \mathbf{E}\left(Z_{y}^{1}\right)+t\right) \leq \exp \left\{-\frac{t^{2}}{2\left|V_{y}\right|\left(y+(\log n)^{2}\right)}\right\} .
$$

Putting $t=n / \delta^{1 / 2}$ and observing that $V_{y} \neq \emptyset$ implies $\delta \leq n^{1 / 4}$ and $y \delta \leq n^{1 / 2}$, and applying (18), (19), (28), we see that

$$
\operatorname{Pr}\left(Z_{y}^{1}>2 \frac{n}{\delta^{1 / 2}}\right) \leq e^{-n^{1 / 2} / 3} .
$$

The lemma follows from (19), (27) and (29).

\section{Proofs of Theorems}

We are now able to prove the Theorems.

Proof of Theorem 1. Let $\Delta \leq n^{1 / 2}$. By Lemma 8, there exists a weight assignment $g: E \rightarrow[w]$ with $m_{g} \leq 6 n / \delta$ and $w=3$. Now by Lemma 4 , $s(G) \leq 3 m_{g} w+m_{g} \leq 60 n / \delta$, proving (b). Similar arguments, using Lemma 7 and Lemma 9 in place of Lemma 8, provide part (a) and (c).

Proof of Theorem 2. The proof is similar to the proof of Theorem 1, just use Lemma 5 in place of Lemma 4.

Proof of Theorem 3. The proof is similar to the proof of Theorem 1, just use Lemma 4 and Lemma 10.

\section{References}

[1] M. Aigner and E. Triesch, Irregular assignments of trees and forests. SIAM J. Discrete Math. 3(1990), no. 4, 439-449.

[2] G. Chartrand and L.Lesniak, Graphs \& Digraphs, Chapman \& Hall, London, 1996. 
[3] G. Chartrand, M.S. Jacobson, J. Lehel, O.R. Oellermann, S. Ruiz, F. Saba, Irregular Networks. Congressus Numerantium 64 (1988), 187 192.

[4] R.J. Faudree and J. Lehel, Bound on the irregularity strength of regular graphs. Colloq. Math. Soc. János Bolyai, 52, Combinatorics, Eger.

North Holland, Amsterdam, 1987, 247-256.

[5] W. Feller, An Introduction to Probability Theory and it's Applications, Wiley, New York, 1950.

[6] S. Janson, T. Łuczak, and A. Ruciński, Random Graphs, Wiley - Interscience Series, New York, 2000.

[7] J. Lehel, Facts and quests on degree irregular assignments, Graph Theory, Combinatorics and Applications, Wiley, New York, 1991, 765-782.

[8] T. Nierhoff, A tight bound on the irregularity strength of graphs. SIAM J. Discrete Math. 13(2000) no. 3, 313-323.

[9] S. Ross, Stochastic Processes, Wiley 1995. 